Historic, Archive Document

Do not assume content reflects current scientific knowledge, policies, or practices. 



\section{TRADE LIST \\ FALL OF 1903}

UIB RAR TREMCENWD * SEP.8 1923 次

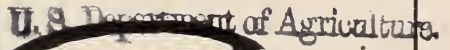

F.U.Meneray Crescent Nursery Company : : : CRESCENT, IOWA Long Distance Telephone Crescent $R=1$ 


\section{TO THE TRADE:}

We take pleasure in presenting our Trade List for Fall, I903.

Extending our thanks for past farors we solicit a continuance of your patronage, and to those with whom we have not had the pleasure of dealing we ask for a trial order.

GUARANTEE.-We exercise the greatest care to have every tree and plant true to name, and are ready, on proper proof, to replace any stock sent out by us that may prove untrue to label, free of charge, or refund the money paid for same. It is understood and agreed between purchasers and ourselves, that we are not to be held liable for any greater sum than that paid us for said trees and plants that prove untrue. 


\section{Terms and Conditions Govern: ing Quotations in Price List}

PACKAGES.-The cost of packages must be added to prices quoted in this list.

P.ACKING.-Goods will be packed in good shape and special care taken to insure their arrival in good condition.

CAUTION.-We accept all orders upon condition that they shall be void should any injury befall the trees and plants from frost. hail or other causes, orer which we haye no control.

TERMS.-Cash. When desired we will allow from 30 to 60 days, from date of shipment, to responsible, prompt paying customers. Parties with whom we have had no business transactions will save time by sending reference with order.

FORMS OF PAYMENT. - Remittances should be made by drafts on New York or Chicago, or by money orders on express companies or postal orders.

INSPECTION.-Our nursery has been inspected by State Entomologist and has been found free from dangerous insects and disease. A copy of certificate of inspection will be attached to every package that leaves our nursery.

\section{F. W. Meneray Crescent Nursery Co. CRESCENT, IOWA}

Location of Nursery-Seren miles north of Council Bluffs, Iowa, on the C. \& N. W. R. R. 


\section{FRUIT DEPARTMENT}

APPLES.

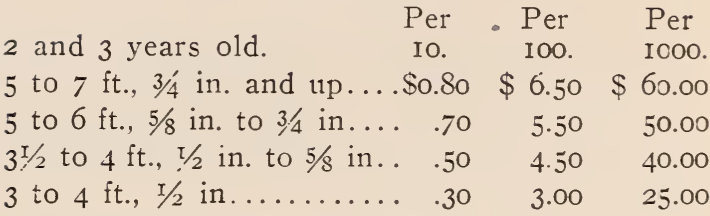

Arkansas Black

Malinda

Antenovka

McMahon

Anisim

Winkler

Allen's Choice

Missouri Pippin

August Sweet ${ }^{\circ}$

Missouri Superior

Blendairs

Babbitt

Blackanette

Black Twig

Borwincka

Blush Caville

Butulen

Chismen

McGee's Pippin

Meneray

Mammoth Black Twig

N. W. Greening

Nebraska Blush

Newport Winter

Porter

Peter

Duchess

Paten's Greening

Duchess No. 4

Duchess No. 6

Dunlap

Pewaukee

Red June

Roman Stem

D. K. G. Peasant

Red Astrachan

Early Vornish

Early Harvest

Early Colton

Everlasting

Red Transparent

Rawls' Jannette

Remna

Rubican

Famuce

Sandy Glass

Fulton

Sheriff

Grimes' Golden

Gano

Scott's Winter

Sievers 
Glass Winter

Gregg

Golden Pippin

Harry Kaump

Hybernal

Hass

Indian

Iowa Blush

Iowa Beauty

Jonathan

Kiersh Anis

Longfield

Limber Twig

Luberka

Loy

Lusorka
Sweet Pearmain

Summer Pearmain

Tetosky

Thaler

Twelve Month

Utter's Red

Van Deman

Viealthy

IV. S. Paradise

White Pippin

Wolf River

Wythe

Wine Sap

Yellow Transparent

York Imperial
CRABS.

Alaska

Centerınial

Freeman's

Hesper Blush

Lemon

Milton

Maiden's Blush
Martha

Pearl

Perfection

Standard

Sulard

Virginia

Whitney

PEACHES.

$$
\begin{array}{ccc}
\text { Per } & \text { Per } & \text { Per } \\
\text { Io. } & \text { IOO. } & \text { Ioco. }
\end{array}
$$

5 to $6 \mathrm{ft}$., II-I6 in. and up. $\$ 0.70 \$ 6.00 \$ 55.00$

4 to $5 \mathrm{ft}$., $5 / 8$ in. to II-I6 in. $.50 \quad 4.50 \quad 40.00$

3 to $4 \mathrm{ft}$., 7 - $\mathrm{I} 6 \mathrm{in}$. to $\mathrm{I} / 2 \mathrm{in.} \quad .30 \quad 3.00 \quad 25.00$

Elberta

Price's Seedling

Smock 
PEARS.

$\begin{array}{ccc}\text { Per } & \text { Per } & \text { Per } \\ \text { Io. } & \text { 100. } & \text { I000. }\end{array}$

4 to $6 \mathrm{ft.,} 5 / 8$ in. to $3 / 4$ in ...\$1.50 \$12.00 $3 \frac{1}{2}$ to $4 \mathrm{ft} ., 1 / 2$ in. to $5 / 8$ in. $.80 \quad 7.00$ Kiffer

Garber

\section{CHERRIES.}

$\begin{array}{ccc}\text { Per } & \text { Per } & \text { Per } \\ \text { IO. } & \text { IOO. } & \text { IOOO. }\end{array}$

4 to 6 it., 2 years, $3 / 4$ in. and up $\ldots \ldots \ldots \ldots \ldots$. $\$ 2.50 \quad \$ 22.00 \quad \$ 200.00$ 4 to $5 \mathrm{ft}$., 2 years, $5 / 8$ in. to

$3 / 4$ in. ........... $2.00 \quad$ I $8.00 \quad$ I80.00

3 to $4 \mathrm{ft} ., 2$ years, $1 / 2 \mathrm{in}$. to

$5 / 8$ in. ............ I.75 I6.00 I50.00

$31 / 2$ to $4 \frac{1}{2} \mathrm{ft}$., I year, II-I6

in. and up......... 2.00 I8.00 I75.0J

3 to $4 \mathrm{ft}$., I year, $5 / 8$ in. to

II-I6 in. .......... I.75 I6.00 I50.00

2 to $3 \mathrm{ft}$., I year, $1 / 2 \mathrm{in}$. to

$5 / 8$ in. ........... I. 50 I2.00 100.00

Dyehnuse

Early Richmond

English Morrello

Large Montmorency
Lutovka

Ostheim

Terry's Early

Wragg

\section{PLUMS.}

$\begin{array}{ccc}\text { Per } & \text { Per } & \text { Per } \\ \text { Io. } & \text { IOO. } & \text { IOoo. }\end{array}$

4 to $5 \mathrm{ft.}, 2$ years, $5 / 8$ in. to

II-I6 in. $\ldots \ldots \ldots \ldots \ldots$ I.75 $\$ 15.00$ \$I 40.00

3 to $4 \mathrm{ft.}, 2$ years, $1 / 2$ in. to

$5 / 8$ in. ............ $1.50 \quad 10.00 \quad 90.00$

3 to $4 \frac{1}{2} \mathrm{ft}$., I year, 7 - 16 in.

to $1 / 2$ in ............ $1.25 \quad 9.00 \quad 80.00$

Chas. Downing

Milton 
De Soto

Forest Garden

Hawkeye

Miner
New Wolf

Wolf

Wyant

IVild Goose

N. B.-We have about 8 ,ooo selected seedlings, Plums; 2 years stem, 3 years root, in the following grades: $3 / 4$ in. and up, $5 / 8$ in. to $3 / 4$ in. $\mathrm{T} / 2$ in. to $5 / 8$ in. The above trees were selected from a block of seedlings, 800,000. They will please you; write for prices.

MULBERRIES.

$\begin{array}{ccc}\text { Per } & \text { Per } & \text { Per } \\ \text { Io. } & \text { IOO. } & \text { IOOO. }\end{array}$

Russian, 6 to 7 ft., $3 / 4$ in.

and up ............ \$1.00. \$9.00

Russian, 4 to $6 \mathrm{ft} ., 5 / 8$ in.

to $3 / 4$ in............. .90 7.50

Russian, 4 to $5 \mathrm{ft}$., $1 / 2$ in.

to $5 / 8$ inf............. $.75 \quad 6.00$

Russian, 3 to 4 ft......... 50 .50 $3.50 \quad 30.00$

GOOSEBERRIES.

Houghton Seedling, 2 yrs.,

No. I ..............

Pearl, I year, No. I.......

$\$ 3.50 \$ 30.00$

Pearl, I year, No. 2....... 5.00

Houghton Layers, layered

in July ..............

I. 00

б́.0o

\section{CURRANTS.}

Victoria, White Dutch, I year, No. I...........

Fays, Prol., North Star, I

$\$ 2.00 \$ 16.00$

year ................

$2.50 \quad 20.00$ 
GRAPES.

$\begin{array}{ccc}\text { Per } & \text { Per } & \text { Per } \\ \text { IO. } & \text { IOO. } & \text { 1000. } \\ & \$ 1.50 & \$ 12.00 \\ & 2.50 & 20.00 \\ & 2.50 & 20.00 \\ & 3.50 & 30.00 \\ & 3.00 & 25.00 \\ & 4.00 & 35.00\end{array}$

Concord No. I, I year....

Concord No. I, 2 years....

Niagara No. I, I year.....

Niagara No. I, 2 years....

Worden No. I, I year....

Worden No. I, 2 years....

BLACKBERRIES.

\section{Per}

Io.

Snyder, sucker plants.....

$\begin{array}{ll}\text { Per } & \text { Per } \\ \text { I0O } & \text { I000. }\end{array}$

RASPBERRIES.

Per
IO.

$\$ 0.75 \$ 6.00$

Eureka and Kansas.......

Per Pet

I00. I000.

$\$ 0.60 \$ 5.50$

MT. CHERRIES.

$\begin{array}{ccc}\text { Per } & \text { Per } & \text { Per } \\ \text { Io. } & \text { IOO. } & \text { IOOO. }\end{array}$

$2 \mathrm{~T} / 2$ to $3^{\mathrm{T} / 2 \mathrm{ft} .,} 3$ years...... $\$ 1.25$ \$10.00 $\$ 80.00$ I $1 / 2$ to $2 \mathrm{I} / 2 \mathrm{ft} ., 2$ years...... $2.80 \quad 7.50 \quad 65.00$ I to $2 \mathrm{ft} ., 2$ years.......... .6o $5.50 \quad 50.00$

ASPARAGUS.

$\begin{array}{ccc}\text { Per } & \text { Per } & \text { Per } \\ \text { IO. } & \text { IOO. } & \text { I000. }\end{array}$

Conover's Col. and Palmet-

to, 2 years............

$\$ 0.60 \$ 4.00$

RHUBARB.

$\begin{array}{ccc}\text { Per } & \text { Per } & \text { Per } \\ \text { Io. } & \text { IOO. } & \text { IOOO. }\end{array}$

Peach, (one of the earliest varieties) single eye....

$\$ 6.00 \$ 50.00$ 


\section{ORNAMENTAL DEPART= $M E N T$}

DECIDUOUS TREES.

$\begin{array}{ccc}\text { Per } & \text { Per } & \text { Per } \\ \text { IO. } & \text { IOO. } & \text { IOOO. }\end{array}$

Chestnut Horse, 8 to I5 in. \$o.50 \$3.00\$20.00 Chestnut Horse, 3-4 ft... I.50 I3.00

Cherry, Cerasus, 5-6 ft... I. 50 .

Cherry, Cerasus, $7-8 \mathrm{ft} \ldots 2.00$ Cherry, Cerasus, io $\mathrm{ft} . \ldots 3.00$ Elms, 3-4 ft.......... .50 3.00 Elms, 5-6 ft............ .75 6.00 Elms, 8-io ft.......... I.50 I3.00 Elms, IO-I2 ft........ $3.00 \quad 25.00$ Elms, I2-I 4 ft........ 5.00 40.00

Judas Tree, $4-5 \mathrm{ft} . . . \ldots$ I.50 Judas Tree, $6 \rightarrow 7 \mathrm{ft} . . . \ldots 2.00$ Maple, Hard, 6 $\rightarrow$ ft..... I.50 I2.00 Maple, Hard, 8-io ft.... 2.00 Maple, Hard, Io--I2 ft ... 2.50 Maple, Norway, 6-7 ft... 2.50 Maple, Norway, 8-9 ft... 3.00 Maple, Soft, 3-4 ft..... .35 Maple, Soft, 4-5 ft..... .40 Maple, Soft, 6-8 ft...... I.oo Maple, Soft, 8-io ft.... 2.00 Maple, Soft, Io-I2 ft.... $3.50 \quad 25.00$ Mountain Ash, European, 5 -6 ft. ........... I.25 I 2.00

Mountain Ash, European,

$$
7-8 \text { ft. .............. } 2.00 \quad \text { I5.00 }
$$

Mountain Ash, Domestica, $3-5$ ft. .......... I.25 Poplar, Carolina, $5-6 \mathrm{ft} . . \quad 75 \quad 7.00 \quad 60.00$ 


\section{Per Per Per}

Poplar, Carolina, 8-io ft. \$I.50 \$I 2.50

Poplar, Carolina, I0-I2 ft. $2.50 \quad 20.00$

Poplar, Carolina, I2-I4 ft. $3.50 \quad 30.00$

Poplar, Carolina, I4-I8 ft. $4.50 \quad 40.00$

Poplar, Giant Silver, 3-4 ft. I.00

Poplar, Giant Silver, 4-5 ft. I.50

Russian Olive, 2-2 $1 / 2$ ft. . I.00 8.00

Russian Olive, 4-5 ft..... I.25

Sycamore, 3-4 ft........ .60 5.00

Sycamore, 5-6 ft........ 1.00 9.00

Sycamore, $7-8 \mathrm{ft} \ldots \ldots \ldots$ I. 50 I2.50

Sycamore, 8-io ft...... 2.50 20.00

Sycamore, IO-I2 ft...... $3.00 \quad 25.00$

Tulip Tree, 4-6 ft....... I.50

White Birch, 2-3 ft..... $80 \quad 7.00$

White Birch, 5-6 ft...... I.25

White Birch, 6-7 ft.... 2.00

IVillows, Brittensis, 3-4 ft. $.60 \quad 5.00$

Willows, Laurifolia, 3-4 ft. $\quad .60 \quad 5.00$

Willows, Regalis, 2-4 ft.. $75 \quad 7.00$

Willows, Thurlow's Weep-

ing, $4-5 \mathrm{ft} \ldots \ldots \ldots \ldots$. I.25

Willows, Thurlow's Weep-

ing, $7-8 \mathrm{ft} \ldots \ldots \ldots$.... 50

ORNAMENTAL SHRUBS.

$\begin{array}{ccc}\text { Per } & \text { Per } & \text { Per } \\ \text { IO. } & \text { IOO. } & \text { IO0O. }\end{array}$

Althea's Assorted, $3 \mathrm{ft} \ldots$... 1.25

Baccharis, 3-4 ft........ I.00 \$8.00

Berberry, P. L., 2 years,

20 in. to 24 in......... $40 \quad 3.50 \quad \$ 32.00$

Berberry, P. L.. 3 years,

$2-3 \mathrm{ft} . \ldots \ldots \ldots \ldots \ldots, \quad .50 \quad 4.00$ 


$\begin{array}{ccl}\text { Per } & \text { Per } & \text { Per } \\ \text { Io. } & \text { Ioo. } & \text { IOoo. }\end{array}$

Berberıy. P. L., 3 years.

$4-5 \mathrm{ft} . \ldots \ldots \ldots \ldots \ldots$. .25

Berberry, Com. Green L.,

4 years, $3-4$ ft....... I.25 \$Io.00

Berberry Thunbergi, 4 years

$2 \mathrm{r} / 2-3$ ft. ........... I.25 10.00

Caryopteis, Masta, 2-3 ft. I. I.00 8.00

Crataegus, Oxya, $3 \mathrm{ft} \ldots . . .1 .25$ I0.00

Calycanthus, 2 ft........ I.0O

Calycanthus, $3 \mathrm{ft} \ldots \ldots \ldots$. I.25

Deutzia Scabra, I5 to 20 in. $.80 \quad 6.50$

Deutzia Scabra, 2-3 ft.... $1.00 \quad 8.00$

Deutzia Scabra, heary

clumps, 5-6 ft..... 5.00 40.00 Euonymus, (Strawberry

Tree) $2-3 \mathrm{ft} \ldots \ldots \ldots .75 \quad 6.00$

Eleagnus Longiped, 20 in. .8o 7.00

Eleagnus Longiped, 2-2 $1 / 2$

ft. ............. I.0O 8.00

Exorchorda Grand, 3-4 ft. 2.00

Fringe, Purple, $I_{5}$ to 20 in. $.80 \quad 6.00$

Fringe, Purple, 2-3 ft.... I.00 8.00

Fringe. Purple. 3-4 ft.... I.25 I0.00

Golden Glow ........... .50 3.50

Golden Elder Clumps. 5-6

ft. $\ldots \ldots \ldots \ldots \ldots \ldots .3 .00$

Ginkgio Bilobi, I5-20 in. I.0o, 8.00 Hydrangeas, Io-I2 in.... .75 6.00 Hydrangeas, I6-24 in.... .90 7.00 Hydrangeas, 2-3 ft...... I.25 I0.00 Hydrangeas. heary plants

$3-4 \mathrm{ft} . \ldots \ldots \ldots \ldots$ I. 50 I 2.50

Honeysuckles, Collosa Ru-

bra, Clumps, 4-5 ft... 2.50 20.00 
Honeysuckles, A $1 \mathrm{~b}$ i a,

Clumps, 4-5 ft......\$2.50 \$20.00

Honeysuckles, A $1 \mathrm{~b}$ i a,

Clumps, ${ }_{5}$ in........ .60 5.00

Honeysuckles, Fragrance,

Clumps, 3-5 ft...... 2.50

Hibiscus, Crimson Eye,

2-3 ft. ............ I.00

Iris, German ............ $50 \quad 4.00$

Lilac, Chinese, Tree, $5 \mathrm{ft}$. I.75

Lilac, Japan, Tree, $5 \mathrm{ft} \ldots 2.00$

Lilac, Japan, Tree, 2 ft.... I.75

Lilac, White, Clump, 5-6

ft. .............. 4.00

Lilac, White, Clump, I8-24

in. $\ldots \ldots \ldots \ldots \ldots \ldots . .75 \quad 6.00$

Lilac, Persian, Clump,

$6 \rightarrow \mathrm{ft} . \ldots \ldots \ldots \ldots, 4.00$

Lilac, Persian, Clump,

$4-5 \mathrm{ft} . \ldots \ldots \ldots \ldots .2 .00 \quad 15.00$

Lilac, Persian, Clump,

$2-3 \mathrm{ft} . \ldots \ldots \ldots \ldots, \quad 80 \quad 6.00$

Lilac, Purple, Clump,

${ }_{5} 5-20$ in. .......... $70 \quad 5.00$

Philadelphus, Lem., 2 ft... $\quad .75 \quad 6.00$

Philadelphus, L e m.,

Clumps, 5-6 ft...... 3.50

Philadelphus, $\mathrm{N}$ a n us,

Clumps, $3 \mathrm{ft} . . . \ldots . .2 .50$

Privet, Cal., 2-3 ft....... .75 5.00

Quince, Japan, I8-20 in.. .756 .00

Spireas, Van Houtti, I5-20

in. $\ldots \ldots \ldots \ldots \ldots \ldots$. $70 \quad 5.00$

Spireas, Van Houtti, 2-3 ft. $\quad .80 \quad 7.00$ 


$\begin{array}{ccc}\text { Per } & \text { Per } & \text { Per } \\ \text { IO. } & \text { IOO. } & \text { IOOO. }\end{array}$

Spireas, Van Houtti,

Clumps, $4 \rightarrow \mathrm{ft} \ldots \ldots . \$ 3.50 \quad \$ 30.00$

Spireas, Rubra, Clumps, 5

years $\ldots \ldots \ldots \ldots \ldots . .63 .00 \quad 25.00$

Spireas, Anthony Watereri,

2 years ............. I.00 7.00

Spireas, Bumalda, 2 years. I.0o

Spireas, Thu m bergi

Clumps, 3 to 4 years.. 2.00 I2.50

Spireas, Thu m ber g i

Clumps, 20 in......... I.25 8.00

Spireas, Prunifolia, 3-4 ft. I.00 9.00

Spireas, Sarbofolia, 3 ft... $\quad .75 \quad 6.00$

Spireas, Sarbofolia, I8-24

in. $\ldots \ldots \ldots \ldots \ldots .6 .60$. 400

Spireas, Opulefolia, Clumps,

7-8 ft. ............ 4.00

Spireas, Collosa Albra,

Io in. .............. .75

Siberian Pea Tree, 5-6 ft. 3.00

Siterian Pea Tree, I8-20

in. ................ .70

Symphoricarpus Rubra

$2-3 \mathrm{ft} . \ldots \ldots \ldots \ldots \ldots, \quad .75$

Symphoricarpus Alba, 2-3

ft. ............... .75

Syringas, Common, 20--30

in. ............... 60 .60

Syringas, Common, 2-3 ft. $\quad .80 \quad 7.00$

Syringas, Common, 3-4 ft. I.00 9.00

Syringas, Com. Clumps,

$4-5 \mathrm{ft} . \ldots \ldots \ldots \ldots .3 .50 \quad 25.00$

Syringas, Sweet Scented

Clumps, $4-7 \mathrm{ft} \ldots \ldots .4 .00 \quad 35.00$ 


\section{Per Per Per}

ro. I00. 1000 .

Syringas, Golden Leaf

Clumps, 3-4 ft.....\$2.50

Snowball, 12-r6 in....... .70 $\$ 6.00$

Snowball, I8-24 in....... .90 8.00

Snowball, 2t/2-3 $3 / 2$ ft...... 1.25 I 1.00

Tamrix, Odessana, $12-18$

in. $\ldots \ldots \ldots \ldots \ldots . .50 \quad 3.50$

Tamrix, Odessana, $2 y^{\prime}--5$ ft. $.70 \quad 5.00$

Tamrix, Odessana, $5-7$ ft. $1.00 \quad 7.00$

Weigelia Asst., 20-30 in.. $80 \quad 7.00$

\section{CLIMBERS.}

$\begin{array}{ccc}\text { Per } & \text { Per } & \text { Per } \\ \text { ro. } & \text { Ioo. } & \text { Icoo. }\end{array}$

Honeysuckle, Hall's Japan.\$0.75 \$6.00 Honeysuckle, Belgica .... .75

Trumpet Vine ......... .60 4.00

Wistaria, Fruiteas, 4 years. $1.25 \quad 10.00$

Wistaria, Common Purple. $60 \quad 5.00$

Peony, Single Eye, divided I.25 10.00

Grandifloria

Mrs. Douglas

Mrs. Fletcher

Oliver Logan

Plenissima Rosea

Unnamed Varieties ....... 8 .80 $\quad 7.00 \quad \$ 65.00$

Fifty other named varieties. Prices on application.

ROSES.

$\begin{array}{ccc}\text { Per } & \text { Per } & \text { Per } \\ \text { Io. } & \text { Ioo. } & \text { I000. }\end{array}$

Rosea Rugosa ........\$r.25 \$10.00

Yucca Fil., 2 years....... .40 3.50

Yucca Fil., 4 years....... .60 5.00 


\section{EVERGREEN.}

$\begin{array}{ccc}\text { Per } & \text { Per } & \text { Per } \\ \text { ro. } & \text { roo. } & \text { rooo. }\end{array}$

Colo. Blue Spruce, $2-3 \mathrm{ft} . \$ 10.00$ Colo. Blue Spruce, 3-4 ft. I2.50 Colo. Blue Spruce, 5-6 ft. I5.00 Colo. Silver Bright, 2 ft. . I2.00 Colo. Silver Bright, 3 ft. . I5.00 Colo. Silver Bright, $4 \mathrm{ft} . .20 .00$ Picea Concolor, $3 \mathrm{ft} . \ldots$. I2.00 Picea Concolor, $4 \mathrm{ft} \ldots$... I 5.00 Picea Concolor, $5 \mathrm{ft} . . . .20 .00$

\section{SEEDLINGS.}

Per Per

IOO. $\quad$ IOOO.

Butternut, 8-io in ..........\$r.00 \$8.00 Butternut, I2-I 8 in ......... I.50 I0.0 Black Locust, 6-io in.......... Black Locust, I2--I6 in......... Black I.ocust, I6-24 in........ 2.00 Black Lبocust, $2-3 \mathrm{ft} \ldots \ldots \ldots \ldots \ldots . \ldots$

Catalpa, $6-8$ in............

Catalpa, ro-i6 in............ 2.00

Catalpa, $18-24$ in............ 2.50

Cherrv, Black, 8 - I6 in........

Walnuts, Black, 8-i2 in........ 5.00

Walnuts, Black, I4-20 in....... 6.00

Walnuts, Black, 2 years trans., $20-30$ in. ............. $\quad$ I5.00

Walnuts, English, 2 years trans., $\mathrm{I} 2-\mathrm{I} 8$ in. ............. 6.00

Cottonwood, $8-\mathrm{r} 2$ in..........

Cottonwood, $14-20$ in ......... 1.25

Cottonwood, $20-30$ in......... 


\section{CUTTINGS AND SCIONS.}

\section{Per}

Per

IOO.

Apple Scions-See Apple List for

varieties

Cherry Scions-See Cherry List for

varieties

Plum Scions-See Plum List for

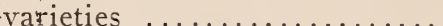

Plum Cuttings, Mariana.............

Cuttings, Currant, Victoria........

Cuttings, Grape, Concord..........

Cuttings, Grape, Champion........

Cuttings, Grape, Brighten.

Cuttings, Grape, Niagara.........

Cuttings, Grape, Worden.

I000.

Special prices on large amount.

Cuttings, Willow. Brittensis...... \$0.50

Cuttings, Willow, Regalis........ .75

Cuttings. Willow. Lorifolia........ .50

Cuttings, Willow, Thurlow's Weep-

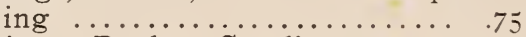

Cuttings, Poplar, Carolina........

Cuttings, Poplar, Giant......... .50

Cuttings, Ornamentals, Deutzia

Scabra .................. .75

Cuttings, Ornamentals, Honeysuckles. Asst. ............. $\quad .75$

Cuttings, Ornamentals, Syringas,

Asst. .................. .50

Cuttings, Ornementals, Spirea Van

Houtti

Cuttings, Ornamentals, Tamrix

$\because$ Odessana

.75

5.00

5.00

N. B.-All stock listed is of our own growing. We are not the largest growers, but we can say that our stock is well grown and every tree and plant is at home upon our grounds. Send us your list; we can please you.

Would be pleased to receive a trial order from you. 